\title{
Optimum Channel Allocation in OFDMA Multi-cell Systems
}

\author{
Andrea Abrardo ${ }^{1}$, Paolo Detti ${ }^{1}$, Gaia Nicosia ${ }^{2}$, \\ Andrea Pacifici ${ }^{3}$, and Mara Servilio ${ }^{4}$ \\ 1 Università degli Studi di Siena, \\ Dipartimento di Ingegneria dell'Informazione, Siena, Italia \\ \{abrardo,detti\}@dii.unisi.it \\ 2 Università degli Studi Roma Tre, \\ Dipartimento di Informatica e Automazione, Roma, Italia \\ nicosia@dia.uniroma3.it \\ 3 Università degli Studi Tor Vergata, \\ Dipartimento di Ingegneria dell'Impresa, Roma, Italia \\ pacifici@disp.uniroma2.it \\ 4 Università degli Studi di L'Aquila, \\ Dipartimento di Informatica, via Vetoio, I-67010 Coppito, L'Aquila, Italia \\ servilio@di.univaq.it
}

\begin{abstract}
This paper addresses the problem of allocating users to radio resources (i.e., sub-carriers) in the downlink of an OFDMA cellular system. We consider a classical multi-cellular environment with a realistic interference model and a margin adaptive approach, i.e., we aim at minimizing total transmission power while maintaining a certain given rate for each user. We discuss computational complexity issues of the resulting model and present a heuristic approach that finds optima under suitable conditions, or "reasonably good" solutions in the general case. Computational experiences show that, in a comparison with a commercial state-of-the-art optimization solver, our algorithm is quite effective in terms of both infeasibilities and transmitted powers and extremely efficient in terms of CPU times.
\end{abstract}

Keywords: Radio resource allocation, network flow models, heuristic algorithms.

\section{Introduction}

IEEE 802.16 Air Interface Standard [6], which is the basis of the WiMAX technology, is the most recent solution for the provision of fixed broadband wireless services in a wide geographical scale and proved to be a real effective solution for the establishment of wireless metropolitan area networks (WirelessMAN). The most flexible physical layer implementation provided by the IEEE $802.16 \mathrm{e}$ standard is the OFDMA. It provides a sub-channelization structure with variable FFT (Fast Fourier Transform) sizes accommodating different channel bandwidths. The active sub-carriers are divided into subsets called sub-channels,

E. Altman and A. Chaintreau (Eds.): NET-COOP 2008, LNCS 5425, pp. 103-111, 2009.

(C) Springer-Verlag Berlin Heidelberg 2009 
being one of the basic units of resource allocation. In OFDMA systems, the concept of multi-user and single-user diversities are strictly connected. Indeed, for fixed or portable applications where the radio channels are slowly varying, an intrinsic advantage of OFDMA over other multiple access methods is its capability to exploit the multi-user diversity embedded in diverse frequency-selective channels 29]. Assigning the available sub carriers to the active users in an adaptive manner is a viable method to achieve multi-user diversity: the propagation channels are independent for each user and thus the sub carriers that are in a deep fade for one user may be good ones for another. Several papers have recently focused on the problem of optimum channel allocation of OFDMA cellular systems, and some of them have also considered the joint scheduling-allocation problem 1012. In general, resource allocation and scheduling tasks consist of either minimizing a cost measure (e.g. transmit power [510]) or maximizing a benefit (e.g. throughput [7/811]) while considering system hardware constraints, service specific quality of service (QoS) requirements and the overall system state. Assuming that the transmitter knows the instantaneous channel state of all users, significant performance improvements can be achieved in OFDMA if the sub-carrier, modulation, coding and power management is performed in an adaptive channel aware manner.

Most of the above studies have so far concentrated on the single cell scenario, where interference among users can be easily avoided by orthogonal assignments. In this context, it is shown that a high multi-user diversity gain can be obtained by means of cross layer approach, even in presence of a high degree of single user diversity. An interesting approach for considering more realistic interference model is considered in [1, where a classical cellular environment is studied. In [1], a realistic interference model is considered, where the ability of two nodes to communicate reliably depends not only on their distance but also on actual interference level which is produced by other users which are assigned the same resource, i.e., the same sub-carrier.

The paper is organized as follows. In Section 2, the problem is formally defined and its complexity is addressed. In Section 3. we proposed a heuristic algorithm for solving the radio resource allocation problem, based on a network flow model, which provides an optimal solution for a simplified version of the problem. Finally, computational results are presented and discussed in Section 4.

\section{Problem Statement}

In this section we describe the interference model used throughout the paper and give a formal statement of the optimization problem that we address.

We are given $(i)$ a set of sub-carriers or radio resources $\{1, \ldots, m\} ;(i i)$ a set of cells $\left\{C_{1}, \ldots, C_{k}\right\}$, and $(i i i)$ for each cell $C_{h}$ a set of $n_{h}$ users $U_{h}=\left\{1, \ldots, n_{h}\right\}$. For each user $i$, we denote by $b(i)$ the cell $i$ belongs to. Hence, $b(i)=C_{h}$ for all $i \in U_{h}$. If we set a certain target spectral efficiency $\eta_{i}$ for user $i$, the transmission requirements correspond to a certain number of sub-carriers $r_{i}=R_{i} / \eta_{i}$, where $R_{i}$ is the transmission rate required by user $i$, and $\eta_{i}$ is set in a such a way that $r_{i}$ is integer. 
In general, users belonging to different cells can share the same sub-carrier, while interference phenomena do not allow two users in the same cell to transmit on the same sub-carrier. However, the power required for the transmission on a given sub-carrier increases with the number of users transmitting on that subcarrier. More precisely, let $S_{j}$ be the set of users which are assigned to (i.e., that are transmitting on) the same sub-carrier $j$. The transmission powers $p_{i}(j)$ requested by users in $S_{j}$ on sub-carrier $j$ satisfy the following system.

$$
\begin{aligned}
& p_{i}(j)=A_{i}(j)+\sum_{\substack{\ell \in S_{j} \\
\ell \neq i}} B_{i}^{b(\ell)}(j) p_{\ell}(j) \quad i \in S_{j} \\
& p_{i}(j) \geq 0 \quad i \in S_{j}
\end{aligned}
$$

where $A_{i}(j)$ and $B_{i}^{b(\ell)}(j)$ are given data taking into account the target signalinterference-ratio $\left(S I R_{i}\right)$, the channel gain of user $i$ on sub-carrier $j\left(G_{i}(j)\right)$, and the channel gain between user $i$ and the base station of cell $h \neq b(i)$ on sub-carrier $j\left(G_{i}^{b(h)}(j)\right)$. More precisely, $A_{i}(j)$ is proportional to $S I R_{i} / G_{i}(j)$ and $B_{i}^{b(\ell)}(j)=S I R_{i} G_{i}^{b(\ell)}(j) / G_{i}(j)$. In the following, we refer to the quantities $A_{i}(j)$ and $\sum_{h \in S_{j}, h \neq i} B_{i}^{b(h)}(j) p_{h}(j)$ of System (11) as fixed costs and variable costs, respectively. Note that, System (1) may not have a feasible solution.

A feasible radio resource allocation consists in assigning sub-carriers to users in such a way that $(i)$ for each user $i, r_{i}$ radio resources are assigned to it, (ii) users in the same cell are not assigned to the same radio resource, (iii) given the set $S_{j}$ of users assigned to radio resource $j$, System (11) has a feasible solution, for any sub-carrier $j=1, \ldots, m$. Clearly, a necessary condition for a feasible allocation to exist is $m \geq \max _{h=1, \ldots, k} \sum_{i \in U_{h}} r_{i}$. The problem, that we call Cellular Radio Resource Allocation (RAP), consists of finding a feasible radio resource allocation that minimizes the total transmission power, i.e., the sum of the transmission powers required by all the users. Iteratively solving instances of RAP, on a radio-frame basis, may be used for dynamically assigning radio resources. In fact, practically a carrier is allocated to different users over time.

In Table 1, we summarize some results about the computational complexity for some special cases of the problem. All the details of the polynomial time algorithms and the NP-completeness proofs are reported in [4].

Table 1. Computational complexity results

\begin{tabular}{ccc}
\hline Cells & Special Features & Complexity \\
\hline 2 & cost as in (1) & open \\
2 & identical resources & polynomial (Perfect Matching) \\
fixed $k$ & indistinguishable users & polynomial (min cost flow) \\
3 & identical resources & strongly NP-hard \\
- & identical $A_{i}(j) \forall i, j$ & (from 3-dim axial assignment, [4]) \\
- & convex variable costs (Sect. 3) & strongly NP-hard (from 3-dim matching) \\
- & limited cell power & strongly NP-hard (from 3-Partition, [1]) \\
\hline
\end{tabular}


In the first column of the table, the number of cells of the system is indicated, while the second column reports the special characteristics of the problems. In the problem with "identical resources", the fixed and variable costs do not depend on the particular resource the users are assigned to. The term "indistinguishable users" refers to the case in which the transmission power on a resource depends only on the number of users transmitting on it, and by the cell the users belong to. The case with convex variable costs is thoroughly addressed in Section 3. In the last row of the table, "Limited cell power" means that an upper bound on the transmission power of the users in the same cell is given. In this latter case, NP-hardness holds even if $n_{h}=1$ for any cell $h=1, \ldots, k$ and identical values of the $B_{i}^{h}(j)$ 's.

\section{A Network Flow Based Algorithm}

In this section we propose a heuristic algorithm for solving RAP which is based on a minimum cost flow problem. To this purpose, we exploit a procedure that exactly solves a simplified version of RAP in which the transmission power of any sub-carrier $j$ assumes a special structure.

\subsection{A Simplified Model}

Given a sub-carrier $j$ and a set of users $S$ assigned to it, suppose that the transmission power $T(j)=\sum_{i \in S} p_{i}(j)$ has the following special form:

$$
T(j)=g(|S|)+\sum_{i \in S} A_{i}(j)
$$

where $g(\cdot)$ is a convex function. Then $T(j)$ is comprised by the (usual) fixed cost part, which depends on the set $S$ of users assigned to $j$, plus a convex variable cost which only depends on the number $|S|$ of users assigned to resource $j$. When the transmission power on resource $j$ is expressed as in Equation (2) for all $j=1,2, \ldots, m$, we may find an optimal solution of RAP with the network flow model described hereafter.

Consider the marginal variable cost $\Delta_{\ell}(j)=g(\ell)-g(\ell-1)$ corresponding to assigning an additional user to resource $j$ when $\ell-1 \geq 1$ users have been already assigned to it (let $\Delta_{1}(j)=0$ ). Since $g$ is convex, $\Delta_{\ell+1}(j) \geq \Delta_{\ell}(j) \geq 0$, for all $\ell \geq 1$.

On these grounds, we may define the following flow network $G=(V, A, c)$ illustrated in Figure 1. We distinguish four layers of nodes plus a demand node $t$. The first layer of nodes contains a supply node per each user. We denote the node associated to user $i$ of cell $C_{h}$, as $u_{h}^{i}$. In the second and third layer, for any pair $(j, h)$ associated to resource $j$ and cell $C_{h}$, there is a node $v_{j, h}^{\prime}$ for the second layer and $v_{j, h}^{\prime \prime}$ for the third layer. The fourth layer contains one node for any possible number of users $\ell$ assigned to resource $j$, for all $j \in R$. Since the maximum possible value for $\ell$ equals the number of cells $k$, we have $m k$ nodes in this layer. We refer to the $\ell$-th node corresponding to resource $j$ as $w_{j, \ell}$. The arcs of the network are defined as follows. 


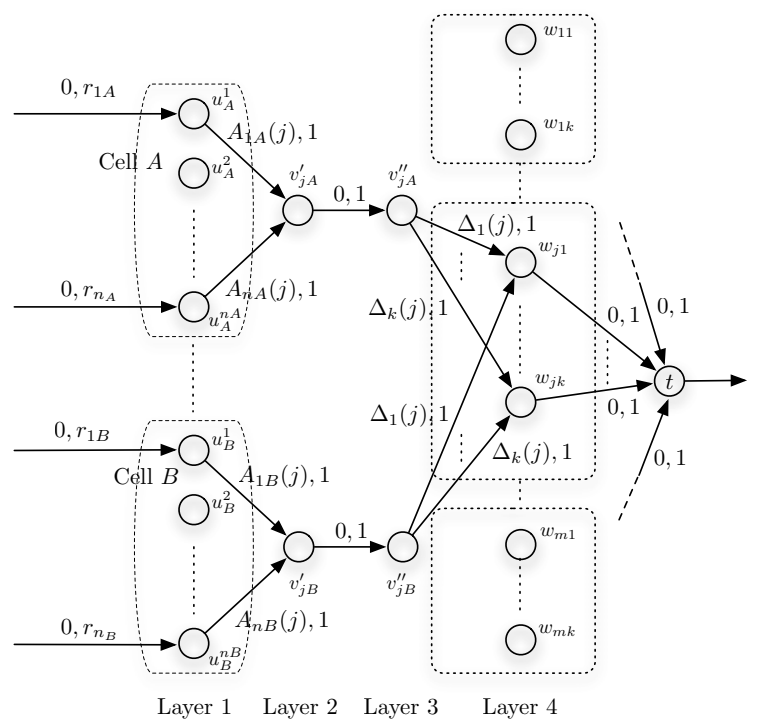

Fig. 1. The network flow model for the simplified RAP

1. For all $j \in R, h \in \mathcal{C}$ and $i \in C_{h}$, arc $\left(u_{h}^{i}, v_{j, h}^{\prime}\right)$, with unit capacity and cost equal to $A_{i}(j)$, connects first and second layer nodes.

2. There is a unit capacity, null cost arc for each pair of nodes $v_{j, h}^{\prime}$ to $v_{j, h}^{\prime \prime}$ between second and third layer.

3. For all $j \in R, C_{h} \in \mathcal{C}$ and $1 \leq \ell \leq k$, unit capacity arc $\left(v_{j, h}^{\prime \prime}, w_{j, \ell}\right)$ connect third and fourth layer nodes. The cost of such an arc is $\Delta_{j}(\ell)$.

4. All the arcs $\left(w_{j, h}, t\right)$ exist, with unit capacity and null cost, for all the nodes $w_{j, h}$ in the fourth layer.

The amount of commodity supplied by each node $u_{h}^{i}$ in the first layer is $r_{i}$, the demand at node $t$ is $\sum_{i} r_{i}$, while all other nodes are neither supply nor demand nodes. It is not hard to prove that there is a one-to-one correspondence between any integer feasible flow on $G$ and a feasible solution of the instance of RAP. Therefore, the minimum cost flow in this supply-demand network provides an optimal solution to the simplified version of RAP.

\subsection{A Heuristic for RAP}

In this section, we propose a heuristic algorithm for RAP based on the network $G=(V, A, c)$ introduced in the previous section. Clearly, the cost structure of RAP does not allow to use the network model introduced above to determine an optimal solution. However, a feasible integer flow on $G$ corresponds to an assignment of users to resources. The idea of the heuristic algorithm is that of approximating the actual costs of RAP with a cost structure satisfying Equation (2) and then applying the network flow model in order to obtain an initial assignment of users to the resources. Note that, such an initial assignment may 
not be feasible in the original problem, since System (10) may not be feasible for some set of users assigned to a resource. In most cases (and as long as a feasible solution of the original instance of RAP exists), possible infeasible assignments can be adjusted, via a local search procedure, to get a feasible solution. Even when feasible assignments exist, the procedure does not guarantee to find one of these solutions. However, as shown in Section 4 numerical experiments give an evidence that the procedure is indeed effective in this regard.

We proceed as follows, to approximate the costs in order to apply the model described in Section 3.1. In order to compute the variable costs part, we set $A_{i}(j)=\bar{A}(j)$ and $B_{i}(j)=\bar{B}(j)$ in System (11) for all $i \in U$ and $j \in R$, thus making the users indistinguishable. The linear system (1) becomes

$$
p_{i}(j)=\bar{A}(j)+\bar{B}(j) \sum_{\substack{h \in S_{j} \\ h \neq i}} p_{h}(j) \quad i \in S_{j}
$$

After some algebra, the total transmission power required on resource $j$ becomes

$$
T_{t}^{\prime}(j)=\sum_{i \in S_{j}} p_{i}(j)=\frac{t \bar{A}(j)}{1-(t-1) \bar{B}(j)}=t \bar{A}(j)+\frac{t \bar{A}(j)(t-1) \bar{B}(j)}{1-(t-1) \bar{B}(j)}
$$

where $t=\left|S_{j}\right|$. Note that $T_{t}^{\prime}(j)$ only depends on the number $t$ of users assigned to $j$. According to the last expression of (44), $T_{t}^{\prime}(j)$ can be decomposed into a fixed part, $t \bar{A}(j)$, and variable cost part, $g_{j}(t)=\frac{t \bar{A}(j)(t-1) \bar{B}(j)}{1-(t-1) \bar{B}(j)}$, as in (2). It is easy to show that $g(\cdot)$ is a convex function in $t$.

In our heuristic, we use the network described in Section 3.1, in which the costs of arcs connecting first and second layer nodes are the actual fixed costs coefficients $A_{i}(j)$, while the costs $\Delta_{\ell}(j)$ of arcs connecting the nodes of third and fourth layers are equal to $g_{j}(\ell)-g_{j}(\ell-1)$. Observe that $g_{j}(t)$ could be negative for some value of $t$. In such cases, we say that it is infeasible to allocate $t$ or more users to resource $j$, and we set $\Delta_{\ell}(j)=+\infty$, for all $\ell \geq t$. Observe that the flow between the second and third layer nodes defines a user-resource assignment and, as already mentioned, a feasible solution of the network flow problem may not correspond to a feasible solution for the original problem. Hence, the correspondence between the feasibility of the solutions of the two problems strictly depends on the choice of $\bar{A}(j)$ and $\bar{B}(j)$ parameters used to compute $g_{j}(\cdot)$.

We are now in the position to give a sketch of a heuristic, called NETWORK, for RAP. The procedure inputs a numerical instance of RAP and iteratively solves the network flow problem described above, as follows:

1. the parameters $\bar{A}(j)$ and $\bar{B}(j)$ are initially set to suitable values;

2. network $G=(V, A, c)$ is built and an optimal solution on $G$, i.e., an assignment $\mathcal{A}$ of users to resources, is found;

3. if an infeasibility is detected in $\mathcal{A}$, say on resource $j$, NETWORK carries out a local search in which, basically, the assignment of two users are exchanged: one user $i$ is chosen in $S_{j}$ and it is substituted by another user $\ell$ of the 
same cell $b(i)$ (thus $\ell \notin S_{j}$ ). This search is performed for a given number of iterations or until all the infeasibilities are removed;

4. if $\mathcal{A}$ is feasible, some of the parameters $\bar{A}(j)$ and $\bar{B}(j)$ are updated, in order to improve the quality of the solution (i.e., the total transmission power). To this purpose, the fixed costs of those users requiring the highest transmission powers in the current assignment are increased.

Steps 1-4 are repeated for a limited number of iterations. Eventually, NETWORK returns an assignment of all the users to the sub-carriers, if a feasible assignment has been found. If this is not the case, i.e., infeasibilities cannot be removed, the procedure returns an assignment of a subset of users (partial assignment). It is worthwhile to mention again that, given that the time required for collecting data and computation times are short enough, NETWORK may be used for dynamically assigning radio resources on a radio-frame basis.

\section{Computational Experiments and Conclusions}

In this section, computational results of the heuristic NETWORK on randomly generated instances are presented. The algorithm has been coded in standard C; CPLEX 9.1 has been used for solving the network flow problems. The performances of the heuristic have been compared with a truncated branch and bound algorithm that uses an Integer Linear Programming formulation solved with CPLEX 9.1 (most of the solutions are, in fact, optima or near-optimal solutions). In the instances, the number of cells is $K=7$, the cell radius is $500 \mathrm{~m}$, and the overall signal bandwidth is $B_{\text {tot }}=5 \mathrm{MHz}$. We assume a fixed throughput per cell equal to $R_{t o t}$ bit/s and that such a per-cell throughput is evenly shared among $\left|U_{k}\right|=n_{k}$ users, which are uniformly distributed in hexagonal cells of radius $R$. In all the instances, $m=16$ sub-bands, each with a bandwidth of $B=312.5 \mathrm{kHz}$, and $n_{k}=4$ users per cell have been considered. Hence, each user has a target throughput of $R_{\text {tot }} / 4$ bit/s. As in [1], we assume that all users adopt the same transmission format, i.e., $\eta_{i}=\eta$ for all users on all sub-carriers. Since the rate per sub-carrier is $B \eta$, the condition to achieve the requested $R_{t o t}$ is that $\eta=\frac{R_{t o t}}{16 \times B}$. Note that, in this case, each user is assigned a fixed number of sub-carriers $r_{i}=4$. In all the instances $R_{t o t}=B_{t o t} \times \eta$. Hence, the bigger is $\eta$ the bigger is the cell (fixed) target throughput. Three scenarios have been considered $(\eta=2,2.5,3.0)$ each corresponding to one hundred instances.

Table 2 illustrates a comparison of the results obtained by applying our NETWORK heuristic (columns 2-4) and the CPLEX truncated branch and bound (columns 5-6). Average values of the following data are given: $(i)$ solution, i.e., total transmission power, (ii) computation times in seconds, and ( iii) a measure of rate loss. More precisely, column 3 reports the percentage of resources with infeasible assignments obtained by the NETWORK heuristic. Column 6 reports the number of instances, out of 100, in which the branch and bound cannot find any feasible solution within the time limit (these results have been presented in [1]). In fact, for the infeasible instances, the branch and bound algorithm cannot return an allocation for any user request. Note also that column 4 reports power 
Table 2. Computational results

\begin{tabular}{|c|c|c|c|c|c|c|}
\hline \multirow[b]{2}{*}{$\eta$} & \multicolumn{3}{|c|}{ Network } & \multicolumn{3}{|c|}{ Branch and Bound } \\
\hline & Sol.value & Time $\%$ & Rate loss & Sol.value & Time & \# Infeas. \\
\hline$\overline{2.0}$ & 67.59 & 0.31 & 0.0 & 48.56 & 201.68 & 0 \\
\hline 2.5 & 251.18 & 0.64 & 0.07 & 101.92 & 268.78 & 2 \\
\hline 3.0 & 710.92 & 1.51 & 0.46 & 215.58 & 339.56 & 18 \\
\hline
\end{tabular}

values that are indeed computed over the set of instances for which a feasible solution exists. In most cases, NETWORK can find a feasible solution of reasonable quality in less than one second (which is less than one hundredth than the time required by the branch and bound.) This proves that an implementation of our algorithm on a dedicated processor is suitable for usage in an iterative scheme that solves the problem dynamically.

The power values (at least for the first scenario with $\eta=2.0$ ) obtained by applying NETWORK, exceed those of the branch and bound by about $28 \%$ over all the 100 instances. However, if we compare the results, restricting our observations to the best (i.e., less congested) 85 and 70 instances, the gap decreases to $17 \%$ and $11 \%$, respectively.

Possible future directions of research include $(i)$ computational complexity characterization of "open" special cases of RAP; $(i i)$ improvement of the proposed heuristic via the more effective local search scheme; (iii) design of distributed algorithms for RAP.

\section{References}

1. Abrardo, A., Alessio, A., Detti, P., Moretti, M.: Radio resource allocation problems for OFDMA cellular systems. Computers \& Operations Research 36(5), 1572-1581 (2009)

2. Bender, P., Black, P., Grob, M., Padovani, R., Sindhushayana, N., Viterbi, A.: CDMA/HDR: a bandwidth-efficient high-speed wireless data service for nomadic users. IEEE Communications Magazine 38(7), 70-77 (2000)

3. Burkard, R.E., Rudolf, R., Woeginger, G.J.: Three-dimensional axial assignments problems with decomposable cost coefficients. Discrete Applied Mathematics 65, 123-139 (1996)

4. Detti, P., Nicosia, G., Pacifici, A., Servilio, M.: Optimal power control in OFDMA cellular networks. Tech. Report n. 2008-4, Dipartimento di Ingegneria dell'Informazione, University of Siena (2008)

5. Ergen, M., Coleri, S., Varaiya, P.: QoS aware adaptive resource allocation techniques. for fair scheduling in OFDMA based broadband wireless systems. IEEE Transactions on Broadcasting 49(4) (2003)

6. IEEE Standard for Local and Metropolitan Area Networks. Part 16: Air Interface for Fixed Broadband Wireless Access Systems, IEEE 802.16-2004 revision

7. Pietrzyk, S., Jannsen, G.: Multiuser subcarrier allocation for QoS provision in OFDMA systems. In: IEEE Vehicular Technology Conference, VTC (2002) 
8. Rhee, W., Cioffi, J.M.: Increase in capacity of multiuser OFDM system using dynamic subchannel allocation. In: Proc. IEEE Vehicular Technology Conference, VTC (2000)

9. Shakkottai, S., Rappaport, T.S., Karlsson, P.C.: Cross-Layer Design for Wireless Networks. IEEE Communications Magazine, 41(10) (2003)

10. Wong, C., Cheng, R., Lataief, K., Murch, R.: Multiuser OFDM with adaptive subcarrier, bit and power allocation. IEEE Journal on Selected Areas in Communications 17(10), 1747-1758 (1999)

11. Yin, H., Liu, H.: An efficient multiuser loading algorithm for OFDM based broadband wireless systems IEEE Globecom (2000)

12. Zhang, Y., Letaief, K.: Energy-efficient MAC-PHY resource management with guaranted QoS in wireless OFDM networks. In: Proc. IEEE ICC, Seoul, Korea (2005) 